\title{
RESINA DE Protium heptaphyllum: ISOLAMENTO, CARACTERIZAÇÃO ESTRUTURAL E AVALIAÇÃO DAS PROPRIEDADES TÉRMICAS
}

Gerardo Magela Vieira Júnior, Cleide Maria Leite de Souza e Mariana Helena Chaves*

Departamento de Química, Universidade Federal do Piauí, 64049-550 Teresina - PI

Recebido em 19/9/03; aceito em 29/10/04; publicado na web em 28/1/05

\begin{abstract}
THE Protium heptaphyllum RESIN: ISOLATION, STRUCTURAL CHARACTERIZATION AND EVALUATION OF THERMAL PROPERTIES. Three mixtures of triterpenes (maniladiol and breine; $\alpha$ and $\beta$-amyrin; lupenone, $\alpha$ and $\beta$-amyrinone) were isolated from Protium heptaphyllum March resin. The structural identification was based on NMR and mass spectrometry data. Lupenone, and $\alpha$ and $\beta$-amyrinone were not reported before as constituents of this resin. The resin was submitted to methylation and acetylation reactions. The pure and derivatized resins and the mixtures (maniladiol and breine; $\alpha$ and $\beta$-amyrin) were analyzed by TG and DSC. The TG curves revealed that the derivatization decreases the thermal stability of the resin. The DSC curves showed peaks that can be assigned to evaporation and phase transitions processes.
\end{abstract}

Keywords: Burseraceae; triterpenes; thermal analysis.

\section{INTRODUÇÃO}

O estudo de produtos naturais têm avançado bastante nos últimos tempos em função do desenvolvimento de novas técnicas espectroscópicas e cromatográficas. O isolamento dessas substâncias naturais fornece matéria-prima importante para o preparo de diversos produtos industrializados, o que requer o conhecimento de suas propriedades físicas e químicas. As propriedades térmicas das substâncias, tais como as transições de fase e processos de degradação térmica, têm um papel decisivo na qualidade do produto final, podendo ser convenientemente estudadas por termogravimetria (TG) e calorimetria exploratória diferencial (DSC) ${ }^{1}$.

A espécie Protium heptaphyllum March (Burseraceae), conhecida popularmente como almécega, é encontrada na região Amazônica, no Piauí, na Bahia, em Minas Gerais e Goiás, e em países como Suriname, Colômbia, Venezuela e Paraguai ${ }^{2}$. Esta espécie exsuda uma resina oleosa e amorfa, cujas aplicações gerais vão desde a fabricação de vernizes e tintas, na calafetagem de embarcações, em cosméticos e em repelentes de insetos. Apresenta algumas indicações terapêuticas, como cicatrizante e expectorante e as ações antiulcerogênica e antiinflamatória já comprovadas ${ }^{3,4}$.

A resina da almécega é constituída por substâncias de natureza terpênica, sendo o óleo essencial rico em monoterpenóides e fenilpropanóides ${ }^{4,5}$. Entre os constituintes fixos, a literatura registra a presença de um monoterpeno trioxigenado e quatro misturas binárias de triterpenóides ${ }^{3,6}$.

No presente trabalho, foram isoladas três misturas, totalizando sete triterpenos pentacíclicos (Figura 1), da resina de almécega, identificados como $\alpha$-amirina (1a), $\beta$-amirina (2a), breína (3a), maniladiol (4a), $\alpha$-amirinona (5a), $\beta$-amirinona (6a) e lupenona (1b). Reações de acetilação e metilação foram efetuadas com a resina, devido à presença de grupamentos alcóolicos e ácidos em seus constituintes. Curvas termogravimétricas (TG) e de calorimetria exploratória diferencial (DSC) foram obtidas para a resina natural, derivatizada, e para os principais constituintes, com o objetivo de avaliar suas propriedades térmicas e contribuir para a busca de possíveis aplicações tecnológicas deste material.

*e-mail: mariana@ufpi.br

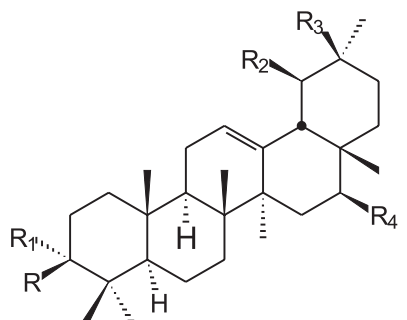

(a)

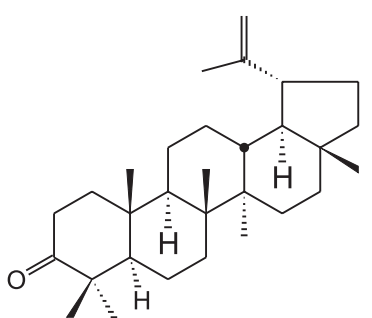

(1b)

$$
\begin{array}{llll}
\mathbf{1 a} & \mathrm{R}=\mathrm{OH} & \mathrm{R}_{1}=\mathrm{R}_{3}=\mathrm{R}_{4}=\mathrm{H} & \mathrm{R}_{2}=\mathrm{CH}_{3} \\
\text { 2a } & \mathrm{R}=\mathrm{OH} & \mathrm{R}_{1}=\mathrm{R}_{2}=\mathrm{R}_{4}=\mathrm{H} & \mathrm{R}_{3}=\mathrm{CH}_{3} \\
\text { 3a } & \mathrm{R}=\mathrm{R}_{4}=\mathrm{OH} & \mathrm{R}_{1}=\mathrm{R}_{3}=\mathrm{H} & \mathrm{R}_{2}=\mathrm{CH}_{3} \\
\text { 4a } & \mathrm{R}=\mathrm{R}_{4}=\mathrm{OH} & \mathrm{R}_{1}=\mathrm{R}_{2}=\mathrm{H} & \mathrm{R}_{3}=\mathrm{CH}_{3} \\
\mathbf{5 a} & \mathrm{R}=\mathrm{R}_{1}=\mathrm{O} & \mathrm{R}_{2}=\mathrm{CH}_{3} & \mathrm{R}_{3}=\mathrm{R}_{4}=\mathrm{H} \\
\mathbf{6 a} & \mathrm{R}=\mathrm{R}_{1}=\mathrm{O} & \mathrm{R}_{2}=\mathrm{R}_{4}=\mathrm{H} & \mathrm{R}_{3}=\mathrm{CH}_{3}
\end{array}
$$

Figura 1. Substâncias isoladas da resina de Protium heptaphyllum: $\alpha$-amirina (1a), $\beta$-amirina (2a), breína (3a), maniladiol (4a), $\alpha$-amirinona (5a), $\beta$ amirinona (6a) e lupenona (1b)

\section{PARTE EXPERIMENTAL}

\section{Procedimentos experimentais}

O fracionamento cromatográfico foi realizado em coluna, à pressão atmosférica, utilizando gel de sílica 60 (0,063-0,200 mm) da Merck. As placas para cromatografia em camada delgada comparativa (CCDC) foram preparadas com $0,25 \mathrm{~mm}$ de espessura, usando como fase estacionária, uma mistura 1:1 de gel de sílica 60 $\mathrm{GF}_{254}$ da Fluka e $60 \mathrm{G}$ Vetec. As revelações das placas cromatográficas foram feitas por borrifamento com solução de sulfato cérico. Os espectros de RMN foram obtidos em espectrômetro Brüker, modelo Avance DRX-500, operando a $500 \mathrm{MHz}$, na frequiência do hidrogênio, e a $125 \mathrm{MHz}$ na freqüência do carbono-13. O solvente utilizado na dissolução das amostras para obtenção dos espectros foi o clorofórmio deuterado $\left(\mathrm{CDCl}_{3}\right.$, Isotec - INC). A análise por CG-EM foi realizada em cromatógrafo da Shimadzu, modelo CG-17A, com coluna capilar DB-1 de $30 \mathrm{~m}$ de comprimento, com 0,25 mm de diâmetro interno e 0,25 $\mu \mathrm{m}$ de espessura 
do filme; temperatura programada da coluna: $60{ }^{\circ} \mathrm{C}$ por $1 \mathrm{~min}$, velocidade de aquecimento: $9,7^{\circ} \mathrm{C} \min ^{-1}$ até $280{ }^{\circ} \mathrm{C}$, mantido por $20 \mathrm{~min}$; temperatura do injetor: $250{ }^{\circ} \mathrm{C}$; temperatura da interface: $315^{\circ} \mathrm{C}$; gás de arraste: hélio, com fluxo de $0,5 \mathrm{~mL} \mathrm{~min}^{-1}$. A corrida com detetor de massas da Shimadzu modelo QP-5000, voltagem do filamento: $70 \mathrm{eV}$; voltagem do detetor: $1,3 \mathrm{KV}$ e analisador do tipo quadrupolo, foi feita na forma scan, com tempo de aquisição de 3 a 57 min e corte do solvente em 8 min; faixa de massas: 35 a 600 .

As curvas TG e DSC foram obtidas nos equipamentos de análise termogravimétrica, TGA 2050, e de calorimetria exploratória diferencial, DSC 2920, ambos da TA Instruments. As análises termogravimétricas (TG) foram realizadas com razão de aquecimento de $10{ }^{\circ} \mathrm{C}$ min $^{-1}$, em atmosfera de $\mathrm{N}_{2}$, sob vazão de $150 \mathrm{~cm}^{3}$ $\mathrm{min}^{-1}$, elevando-se a temperatura de 30 até $500^{\circ} \mathrm{C}$. As curvas DSC foram obtidas com razão de aquecimento de $20{ }^{\circ} \mathrm{C} \mathrm{min}^{-1}$, em atmosfera de $\mathrm{N}_{2}$ com vazão de $50 \mathrm{~cm}^{3} \mathrm{~min}^{-1}$, iniciando o experimento a partir de $30{ }^{\circ} \mathrm{C}$ até $300{ }^{\circ} \mathrm{C}$.

Os pontos de fusão das amostras foram determinados em equipamento da Microquímica, modelo Morf-301. As medidas foram realizadas utilizando razão de aquecimento de $2{ }^{\circ} \mathrm{C} \min ^{-1}$.

\section{Material vegetal}

A resina de almécega foi adquirida em agosto de 2001, no Mercado Central de Teresina - PI, proveniente de um almecegal existente no município de Timom - MA. A espécie vegetal foi coletada nesse local e identificada no Herbário Graziela Barroso da UFPI e registrada a exsicata com o $\mathrm{N}^{\circ} 18.247$.

\section{Extração e fracionamento}

O material vegetal (410 g) foi dissolvido em metanol/diclorometano (4:1), filtrado e o solvente concentrado, obtendo-se $408 \mathrm{~g}$ da resina (RAP). A resina (12 g) foi submetida à cromatografia em coluna de gel de sílica (280 g), empacotada com hexano, utilizando como eluente hexano e acetato de etila, em ordem crescente de polaridade. Foram coletadas 104 frações, de aproximadamente $125 \mathrm{~mL}$, as quais foram concentradas em evaporador rotatório, sob pressão reduzida, e analisadas por cromatografia em camada delgada comparativa de gel de sílica. As frações M-19 (5,43 g) e M33 (1,14 g), eluídas em hexano-AcOEt (9:1) foram submetidas à caracterização, utilizando técnicas espectroscópicas (RMN ${ }^{1} \mathrm{H}$ e ${ }^{13} \mathrm{C}$-BB ${ }^{1} \mathrm{HD}$, e DEPT $135^{\circ}$ ) e de análise térmica (TG e DSC). A fração M-13 (0,15 g), eluída em hexano-AcOEt (95:5), foi caracterizada utilizando-se técnicas espectroscópicas de RMN e massas.

\section{Reações de metilação e acetilação da resina}

A resina $(0,549 \mathrm{~g})$ foi submetida à reação com metanol $(22 \mathrm{~mL})$ em presença de ácido sulfúrico concentrado $(0,3 \mathrm{~mL})$ e refluxo por 5 h. O solvente foi evaporado em evaporador rotatório e o material foi suspenso em éter etílico. Após procedimento usual, obteve-se $0,476 \mathrm{~g}$ de resina metilada (RAM) ${ }^{7}$.

Submeteu-se $2 \mathrm{~g}$ da resina a tratamento com anidrido acético (7 $\mathrm{mL})$ e piridina $(4,9 \mathrm{~mL})$, em presença de dimetilaminopiridina (DMAP), à temperatura ambiente por $12 \mathrm{~h}$, obtendo-se, após procedimento usual, 2,204 g de resina acetilada (RAAC/C) ${ }^{8}$.

$\mathrm{O}$ produto metilado $(0,232 \mathrm{~g})$ foi submetido à acetilação, conforme procedimento anteriormente descrito, obtendo-se $0,270 \mathrm{~g}$ da resina totalmente esterificada (RAE) ${ }^{7,8}$. As reações realizadas foram monitoradas por cromatografia em camada delgada comparativa (CCDC).
A resina natural (RAP) e seus produtos de derivatização foram caracterizados por CCDC, RMN, TG e DSC.

\section{RESULTADOS E DISCUSSÃO}

\section{Análise cromatográfica}

A resina apresenta um odor forte, cor branca, alta solubilidade em diclorometano, sendo parcialmente solúvel em metanol. A análise preliminar por CCDC de gel de sílica confirmou a formação dos produtos metilados, acetilados e esterificados. No cromatograma obtido após a reação de metilação, verificou-se a presença de uma mancha bem definida e com fator de retenção $\left(R_{\mathrm{f}}\right)$ maior que a correspondente aos ácidos existentes na resina, sugerindo a ocorrência desta reação. O cromatograma obtido, com a resina de almécega e o produto resultante da reação de acetilação, apresentou manchas com $\mathrm{R}_{\mathrm{f}}$ bem distintos e a mancha referente ao produto acetilado, menos polar que o álcool correspondente, apresentou maior $\mathrm{R}_{\mathrm{f}}$, comprovando a ocorrência da reação.

\section{Análise espectroscópica}

Os espectros de $\mathrm{RMN}{ }^{1} \mathrm{H}$ da resina não derivatizada, metilada, acetilada, esterificada e das frações M-13, M-19 e M-33 apresentaram um padrão característico de triterpenóides, confirmado pela presença de vários singletos na região de $\delta 0,8$ a 1,3 . No espectro da resina (RAP) observou-se um duplo dubleto em $\delta 3,25$ (J=6 e 11 $\mathrm{Hz}$ ), característico de $\mathrm{H}-3$ em triterpenóides do tipo $3 \beta-\mathrm{OH}$. Os espectros de RMN ${ }^{13} \mathrm{C}-\mathrm{BB}{ }^{1} \mathrm{HD}$ e DEPT $135^{\circ}$ apresentaram um sinal em $\delta 79,3$, atribuído ao carbono carbinólico C-3. Na região de olefinas foram observados dois pares de sinais mais intensos, referentes aos constituintes majoritários, dos quais $\delta 144,7$ (C) e $\delta$ $122,0(\mathrm{CH})$ caracterizam a $\beta$-amirina (2a); $\delta 139,8(\mathrm{C})$ e $\delta 124,6$ $(\mathrm{CH})$ a $\alpha$-amirina (1a), sendo este último o constituinte principal ${ }^{3}$.

$\mathrm{O}$ espectro de $\mathrm{RMN}{ }^{1} \mathrm{H}$ da resina metilada apresentou um singleto em $\delta 3,20$, atribuído à metoxila e seus espectros de RMN ${ }^{13} \mathrm{C}-\mathrm{BB}{ }^{1} \mathrm{HD}$ e DEPT $135^{\circ}$, apresentaram sinais em $\delta 181,4(\mathrm{C}) \mathrm{e}$ $\delta 51,0\left(\mathrm{CH}_{3}\right)$, correspondentes à carbonila e metoxila de éster, respectivamente ${ }^{9}$.

No espectro de $\mathrm{RMN}{ }^{1} \mathrm{H}$ da resina acetilada, evidenciou-se a presença de um singleto em $\delta 2,05$, correspondente ao grupo metílico ligado à carbonila de éster, o que foi confirmado pelos espectros de $\mathrm{RMN}{ }^{13} \mathrm{C}$, por meio dos sinais em $\delta 171,2(\mathrm{C})$, relativo à carbonila do grupo acetato e em $\delta 81,3(\mathrm{CH})$, referente ao $\mathrm{C}-3$ do esqueleto triterpênico. Este último foi desprotegido de 2,3 ppm pelo grupo acetato em relação ao correspondente C-3 hidroxilado $(\delta 79,0)^{10,11}$. Outra evidência da reação de acetilação foi a desproteção de 1,28 ppm observada para o sinal do hidrogênio ligado ao C-3 que no espectro de $\mathrm{RMN}{ }^{1} \mathrm{H}$ da resina não derivatizada e na metilada, se apresentou como um duplo dubleto em $\delta 3,23$ $\left(\mathrm{J}=6\right.$ e $11 \mathrm{~Hz}$ ), enquanto que no espectro de $\mathrm{RMN}{ }^{1} \mathrm{H}$ da resina acetilada este sinal apareceu em $\delta 4,51(\mathrm{dd}, \mathrm{J}=6,0 \text { e } 10 \mathrm{~Hz})^{10}$.

Os espectros de RMN da resina totalmente esterificada, obtida por metilação seguida de acetilação, apresentaram sinais consistentes aos observados nos espectros da resina metilada e acetilada ${ }^{9,10}$.

O espectro de RMN ${ }^{1} \mathrm{H}$ da fração M-19 apresentou um duplo dubleto em $\delta 3,22(\mathrm{~J}=5$ e $11 \mathrm{~Hz})$, característico de triterpenóides do tipo $3 \beta-\mathrm{OH}$ e dois tripletos em $\delta 5,15$ e 5,20, correspondentes à $\mathrm{CH}$ de olefinas. Esta análise foi confirmada pelos espectros de RMN ${ }^{13} \mathrm{C}$ - BB e DEPT $135^{\circ}$. A comparação dos resultados obtidos com os relatados na literatura permitiu identificar os triterpenóides $\alpha$ amirina (1a) e $\beta$-amirina (2a), na razão de 63:37, determinada por comparação dos valores da integração dos sinais da região de 
olefinas $(\delta 5,15$ e 5,20$)$ e do hidrogênio em carbono carbinólico $(\delta$ $3,22 ; \mathrm{H}-3)$, no espectro de $\mathrm{RMN}{ }^{1} \mathrm{H}^{3,12}$. Considerando-se que foram obtidos, após fracionamento cromatográfico, $5,4 \mathrm{~g}(45,3 \%)$ da mistura de $\alpha$-amirina (1a) e $\beta$-amirina (2a), bem como a proporção entre elas, constatou-se que $28,3 \%(3,4 \mathrm{~g})$ da resina corresponde à $\alpha$-amirina e $16,6 \%(2,0 \mathrm{~g})$ à $\beta$-amirina.

$\mathrm{O}$ espectro de $\mathrm{RMN}{ }^{1} \mathrm{H}$ da fração $\mathrm{M}-33$ mostrou-se semelhante ao de M-19, porém a diferença observada foi um segundo duplo dubleto em $\delta 4,22$, correspondente ao hidrogênio em carbono carbinólico, sendo confirmado pelos sinais em $\delta 67,4$ e $\delta 66,4$ de $\mathrm{CH}$ carbinólicos, observados adicionalmente nos espectros de RMN ${ }^{13} \mathrm{C}-\mathrm{BB}{ }^{1} \mathrm{HD}$ e DEPT $135^{\circ}$, atribuíveis ao C-16. Esta análise permitiu identificar uma mistura de triterpenóides diidroxilados com a segunda hidroxila em C-16, denominados breína (3a) e maniladiol (4a), conforme dados da literatura ${ }^{3}$.

$\mathrm{O}$ espectro de RMN ${ }^{1} \mathrm{H}$ da fração M-13 mostrou-se semelhante ao de M-19, contudo não apresentou o sinal referente a H-3. Na região de olefinas apresentou, adicionalmente, dois dubletos em $\delta$ 4,59 e $\delta 4,71$, mantendo entre si integração correspondente a um hidrogênio e constante de acoplamento de $2 \mathrm{~Hz}$, referente a hidrogênios ligados a carbono em ligação dupla gem-dissubstituída. O espectro de RMN ${ }^{13} \mathrm{C}$ - BB confirmou a análise obtida no espectro de $\mathrm{RMN}{ }^{1} \mathrm{H}$, apresentando três sinais em $\delta 218,2$, $\delta$ 218,3 e $\delta$ 218,6 característicos de carbonila em C-3. A análise da região de olefinas permitiu identificar três pares de sinais em $\delta 109,6\left(\mathrm{CH}_{2}\right)$ e $\delta 150,5$ (C), em $\delta 121,9(\mathrm{CH})$ e $\delta 145,6(\mathrm{C})$ e em $\delta 124,6(\mathrm{CH})$ e $\delta 140,1$ (C) que, de acordo com a metodologia para análise de triterpenóides descrita na literatura, possibilitou evidenciar os esqueletos lup20,29-eno, olean-12-eno e ursan-12-eno, respectivamente ${ }^{11}$. Comparando-se a intensidade dos sinais, verificou-se que o primeiro par corresponde ao triterpenóide identificado como lupenona (1b), componente minoritário da mistura, o segundo é relativo à $\beta$ amirinona (6a) e o terceiro, à $\alpha$-amirinona (5a), o componente majoritário. A atribuição dos demais deslocamentos químicos dos sinais observados no espectro $\mathrm{RMN}{ }^{13} \mathrm{C}$ da fração $\mathrm{M}-13$ foi realizada por comparação com os dados da literatura ${ }^{11}$.

O espectro de massas de M-13 foi obtido por injeção em cromatógrafo gasoso acoplado a espectrômetro de massas (GC/ EM). O cromatograma apresentou três sinais com tempos de retenção 32,9, 33,1 e 33,9 min, cujos espectros não constam na biblioteca Wiley 229 disponível no CG/EM. Os espectros de massas do primeiro e do último sinais apresentaram padrão de fragmentação bastante semelhantes, com $\mathrm{m} / \mathrm{z}, 424\left(\mathrm{M}^{+*}, \mathrm{C}_{30} \mathrm{H}_{48} \mathrm{O}\right)$. O pico base com $\mathrm{m} / \mathrm{z} 218$, juntamente com $\mathrm{m} / \mathrm{z}$ 206, em ambos os espectros, originam-se de uma fragmentação típica de retro-Diels-Alder ocorrida no anel $\mathrm{C}$ em esqueletos oleanano e ursano $(\mathrm{m} / \mathrm{z}, 424 \rightarrow \mathrm{m} / \mathrm{z}$ $\left.218, \mathrm{C}_{16} \mathrm{H}_{26}\right)$ e $\left(\mathrm{m} / \mathrm{z}, 424 \rightarrow m / z \text { 206, } \mathrm{C}_{14} \mathrm{H}_{22} \mathrm{O}\right)^{13}$. Esta análise associada aos dados de RMN mostrou que os sinais com tempo de retenção 32,9 e 33,9 correspondem respectivamente à $\beta$ e $\alpha$-amirinona. $\mathrm{O}$ espectro de massas do sinal com tempo de retenção $33,1 \mathrm{~min}$ apresentou um pico com $\mathrm{m} / \mathrm{z}, 424\left(\mathrm{M}^{+\bullet}, \mathrm{C}_{30} \mathrm{H}_{48} \mathrm{O}\right)$ correspondente ao íon molecular, e pico base com $\mathrm{m} / \mathrm{z}, 205$, consistentes com o relatado na literatura para a lupenona ${ }^{13}$.

\section{Análise térmica}

As curvas TG (Figura 2), obtidas para as amostras de resina de almécega (RAP), resina metilada (RAM), resina acetilada (RAAC/ C) e resina esterificada (RAE), exibiram duas etapas de perda de massa. Na primeira, ocorreu uma perda de 5 a $8 \%$ em massa, na faixa de temperatura de 45 a $208{ }^{\circ} \mathrm{C}$, para as amostras RAP, RAM e RAAC/C, a qual pode ser atribuída à liberação de água e de resíduos de solventes orgânicos absorvidos. Para a amostra RAE, a

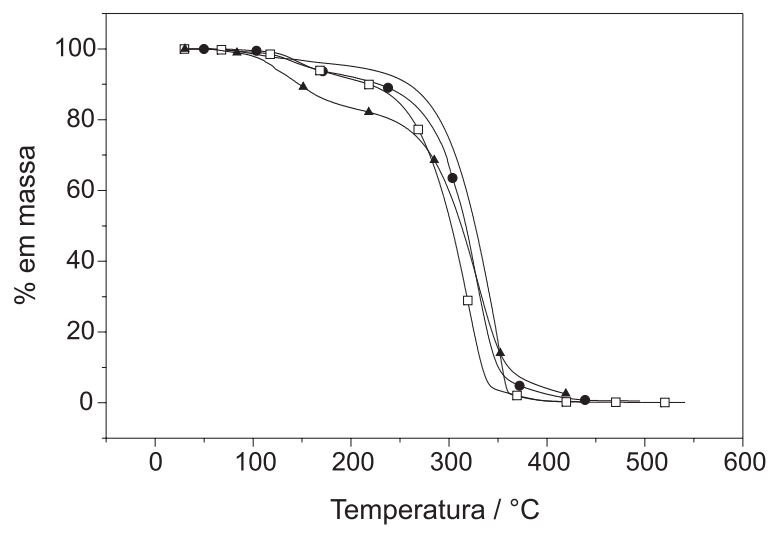

Figura 2. Curvas termogravimétricas para as amostras: (-) resina de almécega pura (RAP), (-—-) resina de almécega acetilada (RAAC/C), (一 - $\square$ resina de almécega metilada $(R A M)$ e (- $\mathbf{\Delta - )}$ resina de almécega esterificada $(R A E)$

curva TG apresentou uma perda de $19 \%$ em massa, na faixa de temperatura de 60 a $225{ }^{\circ} \mathrm{C}$, que pode ser atribuída a liberação de resíduos de solventes e produtos de decomposição. Na segunda etapa, ocorreu a decomposição térmica de todas as amostras, na faixa de temperatura de 205 a $420{ }^{\circ} \mathrm{C}$ para as resinas derivatizadas (RAM, RAAC/C e RAE) e de 210 a $364^{\circ} \mathrm{C}$ para a resina de almécega (RAP). Todas as amostras deixaram resíduos na faixa de 1,5 a 4,0\% em massa. As curvas TG das resinas derivatizadas (Figura 2) indicam uma tendência de decréscimo de estabilidade térmica em relação à resina de almécega (RAP).

Para os constituintes isolados, mistura de $\alpha$ e $\beta$-amirina e mistura dos dióis (breína e maniladiol), as curvas TG (Figura 3) apresentaram uma primeira etapa de perda de massa, atribuída à liberação de resíduos de solventes, na faixa de temperatura de 106 a $208{ }^{\circ} \mathrm{C}$, para a mistura de amirinas e na faixa de 45 a $163{ }^{\circ} \mathrm{C}$, para os dióis. Uma segunda etapa de perda de massa ocorreu na faixa de temperatura de 214 a $320^{\circ} \mathrm{C}$, para as amirinas e na faixa de 240 a $361{ }^{\circ} \mathrm{C}$, para os dióis, deixando resíduos na faixa de 0,5 a $1,5 \%$ em massa. Esta segunda etapa pode ser atribuída principalmente à evaporação das amirinas e dos dióis. Isto explica porque esta etapa se inicia em uma temperatura mais elevada para os dióis, tendo em vista que os mesmos apresentam um grupo $\mathrm{OH}$ a mais que as amirinas, o que implica em forças intermoleculares mais intensas.

A curva DSC (Figura 4) da amostra contendo a mistura de $\alpha$ e $\beta$-amirina apresentou um pico endotérmico em torno de $174{ }^{\circ} \mathrm{C}$, que pode ser associado à fusão destas substâncias, uma vez que esta temperatura foi consistente com a determinada em equipamento de ponto de fusão $\left(171-172{ }^{\circ} \mathrm{C}\right)$.

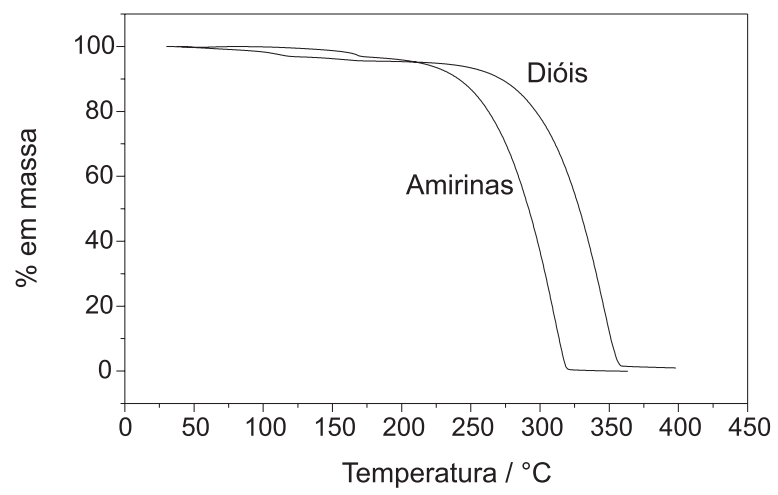

Figura 3. Curvas termogravimétricas para as amostras: $\alpha$ e $\beta$-amirinas $e$ dióis (breína e maniladiol) 


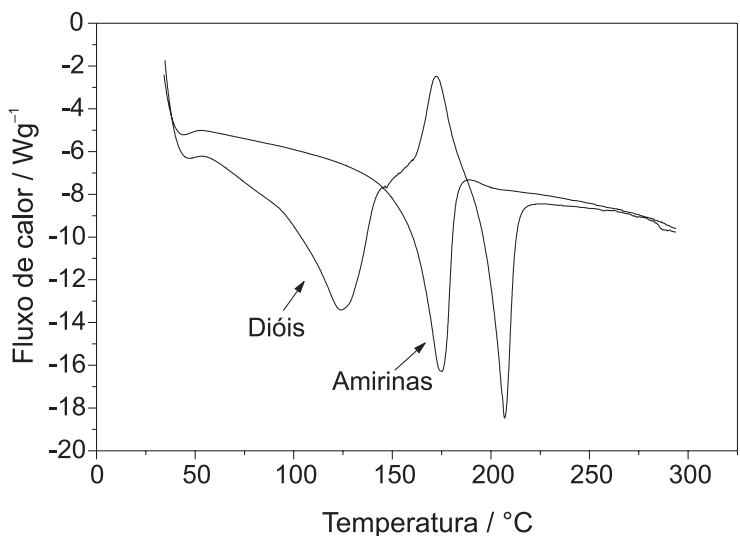

Figura 4. Curva DSC para as amostras: $\alpha$ e $\beta$-amirinas e dióis (breína e maniladiol)

Para a amostra contendo a mistura de dióis (breína e maniladiol), a curva DSC (Figura 4) apresentou um pico endotérmico, entre 85 e $145^{\circ} \mathrm{C}$, que sugere a eliminação de água e resíduos de solventes orgânicos, durante o aquecimento, enquanto que o pico exotérmico, em $172{ }^{\circ} \mathrm{C}$, pode ser decorrente de um processo de cristalização das substâncias. O terceiro evento térmico, por volta de $206{ }^{\circ} \mathrm{C}$, na curva DSC dos dióis, está relacionado à fusão das substâncias, considerando-se que o ponto de fusão do maniladiol reportado na literatura é de $204-205{ }^{\circ} \mathrm{C}^{14}$, sendo também confirmada por medidas de ponto de fusão da amostra $\left(205-206{ }^{\circ} \mathrm{C}\right)$.

Nas curvas DSC (Figura 5) para as amostras RAP e RAM, observou-se a presença de dois eventos endotérmicos. $\mathrm{O}$ primeiro ocorre na faixa de temperatura de 46 a $75{ }^{\circ} \mathrm{C}$, para a amostra RAP, e na faixa de 50 a $104{ }^{\circ} \mathrm{C}$, para a amostra RAM. Este evento pode estar associado à evaporação de substâncias voláteis, tais como resíduos de solventes orgânicos, da mesma forma como sugerido pelas curvas TG (Figura 2). O segundo evento apresenta-se como um pico mais largo, situado entre 104 e $168^{\circ} \mathrm{C}$ para RAP e entre 150 e $250{ }^{\circ} \mathrm{C}$ para RAM. Este evento sugere a evaporação de água. Observa-se um pico endotérmico largo, para a amostra RAP, entre 160 e $200{ }^{\circ} \mathrm{C}$, o qual pode ser atribuído à fusão dos constituintes majoritários da resina, tais como a $\alpha$ e $\beta$-amirinas e os dióis.

As amostras RAAC/C e RAE (Figura 6) apresentaram, cada uma, um pico largo e exotérmico, com máximos em torno de 157 e $143{ }^{\circ} \mathrm{C}$, respectivamente. Estes picos podem estar relacionados à decomposição térmica das amostras.

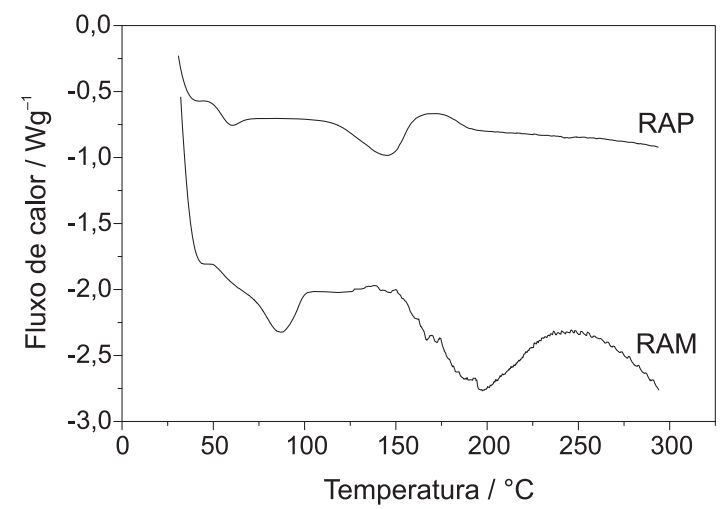

Figura 5. Curva DSC para as amostras: resina de almécega pura $(R A P) e$ resina de almécega metilada (RAM)

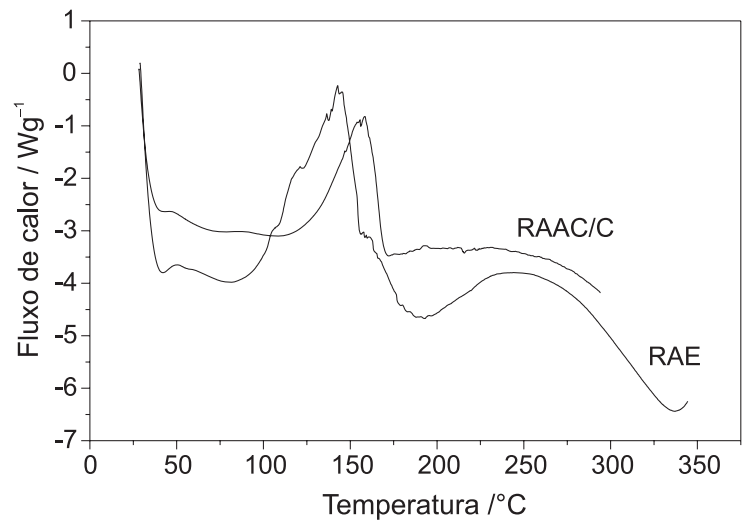

Figura 6. Curva DSC para as amostras: resina de almécega acetilada (RAAC/ C) e resina de almécega esterificada $(R A E)$

\section{CONCLUSÃO}

No estudo da resina da espécie Protium heptaphyllum March, verificou-se uma grande reatividade frente às reações de metilação e acetilação, em decorrência de grupamentos alcoólicos e ácidos presentes em seus constituintes principais. $\mathrm{O}$ fracionamento da resina, por métodos cromatográficos e análise por RMN, permitiu o isolamento e identificação de sete constituintes triterpênicos, distribuídos em três misturas: $\alpha$ e $\beta$-amirina; breína e maniladiol; lupenona, $\alpha$ e $\beta$-amirinona. As substâncias das duas primeiras misturas foram anteriormente relatadas para a resina de almécega, mas os componentes da terceira mistura não tinham sido reportados como constituintes desta resina. Além disso, foi possível verificar que este material se apresenta como uma fonte alternativa para a obtenção de $\alpha$ e $\beta$-amirina em quantidade significativa.

As curvas TG mostraram duas etapas de perda de massa para todas as amostras, sendo a primeira atribuída a processos de evaporação de água, resíduos de solventes orgânicos e a segunda etapa, à degradação da amostra.

A comparação das curvas termogravimétricas mostrou que o processo de derivatização reduz a estabilidade térmica da resina.

As curvas DSC das amostras de resina pura e derivatizadas mostraram eventos endotérmicos característicos de evaporação de substâncias adsorvidas e decomposição térmica, como sugerido nas curvas TG. Para as amirinas e dióis (breína e maniladiol), as curvas DSC sugerem a ocorrência de transições de fase, como fusão e cristalização.

\section{AGRADECIMENTOS}

Os autores agradecem a Profa. Dra. R. F. de M. Barros do Depto. de Biologia - UFPI, pela identificação do material botânico, à CAPES/PROCAD e ao CNPq pelo apoio financeiro e bolsa de Iniciação Científica concedida ao aluno G. M. Vieira Jr., ao Centro Nordestino de Aplicação e Uso da Ressonância Magnética Nuclear (CENAURENM) da UFC pela obtenção dos espectros de RMN, ao Laboratório de Análise de Petróleo (LAPETRO) da UFPI pela permissão para utilização dos equipamentos de análise térmica e CG/EM.

\section{REFERÊNCIAS}

1. Hatakeyama, T.; Quinn, F. X.; Thermal Analysis: fundamentals and applications to polymer science, John Wiley: New York, 1994; Matsuda, A. H.; Machado, L. B.; Mastro, N. L.; Radiat. Phys. Chem. 2002, 63, 353; Macêdo, R. O.; Barbosa-Filho, J. M.; da Costa, E. M.; de Souza, A. G.; J. Therm. Anal. Calorim. 1999, 56, 1353. 
2. Corrêa, M. P.; Dicionário das Plantas Úteis do Brasil, Imprensa Nacional Rio de Janeiro, 1926, vol. 1.

3. Maia, R. M.; Barbosa, P. R.; Cruz, F. G.; Roque, N. F.; Fascio, M.; Quim. Nova 2000, 23, 623; Sussunaga, G. S.; Siani, A. C.; Pizzolatti, M. G.; Yunes, R. A.; Delle Monache, F.; Fitoterapia 2001, 72, 709.

4. Oliveira, F. A.; Vieira-Júnior, G. M.; Chaves, M. H.; Almeida, F. R. C.; Florêncio, M. G.; Lima-Júnior, R. C. P.; Silva, R. M.; Santos, F. A.; Rao, V. S. N.; Pharmacol. Res. 2004, 49, 105; Oliveira, F. A.; Vieira-Júnior, G. M.; Chaves, M. H.; Almeida, F. R. C.; Santos, K. A.; Martins, F. S.; Silva, R. M.; Santos, F. A.; Rao, V. S. N.; Planta Med. 2004, 70, 780; Siani, A. C.; Ramos, M. F. S.; de Lima, O. M.; Santos, R. R.; Ferreira, E. F.; Soares, R. O. A.; Rosas, E. C.; Susunaga, G. S.; Guimarães, A. C.; Zoghbi, M. G. B.; Henriques, M. G. M. O.; J. Ethnopharmacol. 1999, 57, 66.

5. Zoghbi, M. G. B.; Maia, J. G. S.; Luz, A. I. R.; J. Essent. Oil. Res. 1995 7, 541; Bandeira, N. P.; Machado, M. I. L.; Cavalcanti, F. S.; Lemos, T. L. G.; J. Essent. Oil. Res. 2001, 13, 33.
6. Bandeira, P. N.; Pessoa, O. D. L.; Trevisan, M. T. S.; Lemos, T. L. G.; Quim. Nova 2002, 25, 1078

7. Mano, E. B.; Seabra, A. P.; Práticas de Química Orgânica, $3^{\mathrm{a}}$ ed., Edgard Blücher: São Paulo, 1987.

8. Höfle, G.; Steglich, W.; Vorbrüggen, H.; Angew. Chem., Int. Ed. 1978, 17, 569; Chaves, M. H.; Roque, N. F.; Phytochemistry 1997, 44, 523.

9. Fang, J. M.; Wang, K..C.; Cheng, Y. S.; Phytochemistry 1991, 30, 3383.

10. Flagg, M. L.; Valcic, S.; Montenegro, G.; Gomez, M.; Timmermann, B. N.; Phytochemistry 1999, 52, 1345.

11. Olea, R. S. G.; Roque, N. F.; Quim. Nova 1990, 13, 278.

12. Chaves, M. H.; Barbosa, A. S.; Moita Neto, J. M.; Aued-Pimentel, S.; Lago, J. H. G.; Quim. Nova 2004, 27, 404.

13. Budzikiewicz, H.; Wilson, J. M.; Djerassi, C.; J. Am. Chem. Soc. 1963, 85, 3688.

14. Quijano, L.; Rios, T.; Fronczek, F. R.; Fischer, N. H.; Phytochemistry 1998, $49,2065$. 